\title{
Assessment of Fracture Resistance in Teeth with Simulated Perforating Internal Root Resorption Cavities Repaired with Biodentine, Retro MTA and Portland Cement (A Comparative In Vitro Study)
}

\section{Paunceh Samy Soliman ${ }^{1}$, Nehal Nabil Roshdy ${ }^{2 *}$ and Manar Y Fouda ${ }^{3}$}

${ }^{1}$ Master Degree Student, Faculty of Dentistry, Cairo University, Cairo, Egypt

${ }^{2}$ Associate Professor of Endodontics, Faculty of Dentistry, Cairo University, Cairo,

Egypt

${ }^{3}$ Professor of Endodontics, Faculty of Dentistry, Cairo University, Cairo, Egypt
Received: July 07, 2021

Published: July 26, 2021

(C) All rights are reserved by Paunceh Samy

Soliman., et al.

*Corresponding Author: Nehal Nabil Roshdy, Associate Professor of Endodontics,

Faculty of Dentistry, Cairo University, Cairo, Egypt.

\begin{abstract}
Aim: This study was conducted to evaluate the effect of perforation repair materials such as Biodentine, Retro MTA and Portland Cement on the fracture resistance of teeth with simulated perforating internal root resorption cavities.

Methodology: Thirty-five extracted human, single-rooted mandibular premolar teeth were decoronated. Seven teeth were assigned as negative control group. The remaining roots were instrumented with Revo-s rotary files and standardized internal perforating resorption cavities were prepared in the middle third using round burs. Seven teeth were then assigned as a positive control group without any further intervention. The apical third of the remaining root canals were obturated with single-cone technique and divided into 3 experimental groups according to the material used to fill the resorption cavities; Group I: Biodentine, Group II: RetroMTA, Group III: Portland cement and the remaining coronal third of the root canals were backfilled by thermoplasticized gutta percha. For assessment of fracture resistance, teeth were embedded in acrylic resin blocks and subjected to a compressive load via the universal testing machine. The forces at which the fracture occurred were recorded in Newtons and analyzed using One-way ANOVA test followed by Tukey post hoc test for pairwise comparisons, Assessment of the mode of fracture was evaluated using stereomicroscope and data were analyzed using Fisher's exact test.

Results: There was no significant difference found among the three experimental groups ( $p>0.05)$. The Biodentine group showed higher fracture resistance values followed by the Portland cement group and the RetroMTA group respectively with no statistical significant difference among them. The negative control group displayed a significantly higher fracture resistance than all other groups $(p<0.05)$. The mode of fracture was predominately vertical in the Biodentine group and supracrestal in both the RetroMTA and Portland cement group with no statistical significant difference among them ( $p>0.05)$.

Conclusion: Teeth with internal root resorption defects are better filled by a hybrid technique using bioceramic materials such as biodentine in order to enhance the reinforcing capacity of the weakened roots and avoid their fracture.
\end{abstract}

Keywords: Biodentine; Fracture Resistance; Portland Cement; RetroMTA; Root Resorption

\section{Introduction}

Root resorption is either a physiologic or pathologic condition resulting in loss of dentin, cementum and bone. It may occur after various injuries, including mechanical, chemical, or thermal injury [1]. Root resorption can be classified into internal or external root resorption according to the location and site where the resorption 
occur. Internal root resorption (IRR) is a pathologic phenomenon characterized by destruction of intracanal dentine due to odontoclastic activity. This condition is caused by the replacement of normal pulp tissue with granulomatous tissue and giant cell and if internal root resorption is not early detected and remains untreated, it extends and may cause root perforation [2]. Internal root resorption (IRR) with perforation may complicate the prognosis of endodontic treatment because of weakening of the remaining dental structure and this makes the repair process more challenging.

For sealing perforating internal root resorption, several intracanal medicaments have been studied such as calcium hydroxide which has been extensively evaluated and showed well-documented results, however new materials for sealing root perforations have been introduced to the market. These materials are mineral trioxide aggregate and calcium silicate cements (Bioceramics) which possess an antibacterial potential for disinfection of the root canal system and promote healing by mineralized tissue deposition and sealing ability [3].

Mineral Trioxide Aggregate (MTA) exhibits good biocompatibility, bioactivity, osteoconductivity, mechanical strength and provides adequate seal. It is a strong alkaline material that has antibacterial properties and have the ability releasing calcium hydroxide [2].

It has been confirmed that MTA is Portland cement with bismuth oxide added as a radiopacifying agent, which generated an interest in evaluating Portland cement as it has a lower cost than MTA yet having similar physical and biological properties [4].

PC has the main chemical composition, antimicrobial activity and presents biocompatibility similar to MTA. When placed in contact with pulp tissue, MTA and PC provide the same tissue response for direct pulp capping and pulpotomy. Yet, it shows less radioopacity as it lacks the bismuth oxide radioopacifier present in MTA $[5,6]$.

A diversity of novel calcium silicate based biomaterials are presented: such as, RetroMTA, Biodentine, Bioaggregate, EndoSequence and calcium-enriched mixture (CEM) cement [7]. RetroMTA is a new material presented as hydraulic bioceramic, it is a combination of hydrophilic materials in which are not originated from Portland cement. It is recommended by the manufacturer for repairing root resorption and perforations, pulp capping and retro filings. Additionally, manufacturers declared Retro MTA as an aesthetic filling material due to lack of discoloration $[7,8]$.

On the other hand, Biodentine is a new high purity calcium-silicate based dental material demonstrated to have similar or even better features in terms of handling, compressive strength and setting time, while keeping similar or even better sealing properties. Its cytotoxicity is similar to MTA and it has the ability to induce osteoblastic and odontoblastic differentiation of human cultured cells and variously derived human stem cells [9]. Biodentine is recommended for use as a dentin substitute and an endodontic repair material because of its good sealing ability, high compressive strengths, short setting time, biocompatibility, bioactivity and biomineralization properties [10].

To our knowledge, hinted studies were performed to assess the fracture resistance in teeth with simulated perforating internal resorption cavities repaired by Biodentine, Retro MTA and Portland Cement materials. Thus, this study was conducted to evaluate the effect of filling the simulated perforating root resorption defects with these materials on the fracture resistance of the teeth.

\section{Materials and Methods}

Sample size calculation

Based on a previous study by Turker., et al. 2018 [2], Sample size was calculated by using the (G power software) and as regarding the outcome (fracture resistance) we found that 7 teeth per group was appropriate sample size for the study with total sample size 35 teeth ( 5 groups).

The random allocation sequence generation was done using a random sequence number generated by computer software, (http://www.random.org/).

\section{Samples selection}

Thirty five freshly extracted single rooted human mandibular premolar teeth were collected with the Criteria of being non-carious, with straight roots and completely formed apices with the absence of any previous root filling, craze lines, cracks or root resorption. This was verified and confirmed by visual inspection under dental operating microscope. Preoperative radiographs were taken for all teeth with a mesiodistal and buccolingual direction to confirm that they have a single canal with no internal resorption, calcifications or other anomalies.

Teeth were washed under running water and immersed in $5.25 \%$ sodium hypochlorite $(\mathrm{NaOCl})$ solution for 5 minutes to disinfect the teeth and remove any soft deposits on the root surface and an ultrasonic scaler was then used to remove the remaining hard deposits from the root surface. Teeth were then stored in saline $(\mathrm{NaCl} 0.9 \%)$ solution until the time of use. 


\section{Samples preparation}

A total of 35 teeth were decoronated using a low-speed, watercooled diamond disc to obtain a standardized root length of approximately $15 \mathrm{~mm} \pm 1 \mathrm{~mm}$. 7 teeth were then assigned randomly as a negative control group without any further intervention.

The working length of the remaining teeth was established using K-file size 10 to be $1 \mathrm{~mm}$ shorter after the first inspection of the file from the apex and the root canals were instrumented using Revo-S rotary files system up to AS40 (\#40/.06) at rotational speed ranging from 250 - $400 \mathrm{Rpm}$ and torque ranging from $0.8-1.2 \mathrm{Ncm}$ torque according to the manufacturer instructions. The root canals were irrigated after each instrument with $3 \mathrm{ml}$ of $2.5 \% \mathrm{NaOCl}$ for 1 minute.

\section{Resorption cavity simulation}

After preparation, simulated perforating internal resorption cavities were made using a high speed water-cooled size 4 round bur located in the middle third of the distal surface of the roots, 6 - $7 \mathrm{~mm}$ from the coronal cut surfaces (Figure 1A). The canals were irrigated again with $3 \mathrm{ml}$ of $2.5 \% \mathrm{NaOCl}$ for 1 minute followed by 5 $\mathrm{ml}$ of normal saline solution and a final irrigation was applied for 1 minute by using $2 \mathrm{~mL} \mathrm{17 \%} \mathrm{EDTA} \mathrm{to} \mathrm{remove} \mathrm{the} \mathrm{smear} \mathrm{layer,} \mathrm{then}$ the root canals were rinsed with $5 \mathrm{~mL}$ distilled water, dried with paper points and 7 of the samples were then assigned as positive control group without any further intervention.

Apical obturation of the root canals and resorption cavity filling

The apical $5 \mathrm{~mm}$ of the remaining root canals were filled with gutta percha master cones size (\#40/0.04) which match the master apical file with $\mathrm{AH}$ plus sealer using single cone technique. A heated plugger was inserted to partially remove the gutta percha leaving only the apical $5 \mathrm{~mm}$ of the filling just below the perforation cavity. Then the samples were randomly distributed into 3 experimental groups 7 teeth in each according to the filling material used to fill the perforating resorptive defects as mentioned below:

- Group I: Resorption cavities were filled with Biodentine, according to the manufacturer's instructions, 5 drops of the liquid were poured into the powder containing capsule. The capsule was closed and triturated for 30 seconds on a mixing device, Biodentine was carried by MTA carrier and placed incrementally from the coronal cut surface into the resorption cavities and packed using a hand pluggers only up to the resorption cavity level leaving the coronal part of the root canal empty.
- Group II: Resorption cavities were filled with RetroMTA, according to the manufacturers' instructions, Retro MTA was manipulated by mixing $0.3 \mathrm{~g}$ of powder with 3 drops of the liquid for 20 seconds with the use of a plastic spatula (wetting action). Retro MTA was then carried by MTA carrier and placed incrementally from the coronal cut surface into the resorption cavities and packed using a hand pluggers only up to the resorption cavity level leaving the coronal part of the root canal empty.

- Group III: Resorption cavities were filled with Portland cement, Barium sulphate as a radiopacifier was incorporated in the ratio of $25 \%$ by weight into the PC [11]. PC was mixed in a 3:1 powder-distilled water ratio and was carried using MTA carrier and placed incrementally from the coronal cut surface into the resorption cavity and packed using a hand plugger up to the level of the resorption cavity only leaving the coronal part of the root canal empty [12].

Excess material was trimmed from the surface of the teeth using a scalpel and then the teeth were wrapped in a wet gauze, placed in an incubator, and allowed to set for 24 hours at $37^{\circ} \mathrm{Cwith} 100 \%$ humidity before backfilling of the root canals with gutta-percha [2].

\section{Backfilling of the root canals}

Thermoplasticized gutta percha was injected incrementally coronal to the resorption cavities and then packed using hand pluggers and then all specimens were stored for 1 week to allow proper setting of the materials until tested for fracture resistance [2] (Figure 1B).

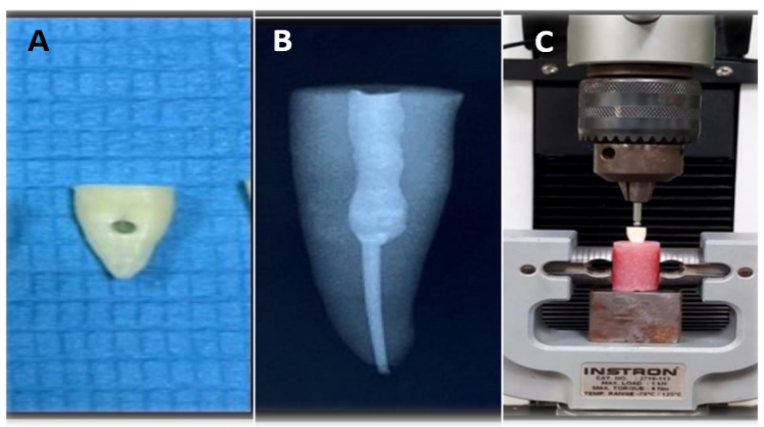

Figure 1: (A) Tooth with simulated perforating internal resorption cavity. (B) A radiographic picture for a sample after filling. (C) A sample placed on the universal testing machine. 


\section{Assessment of fracture resistance}

The apical $5 \mathrm{~mm}$ of all roots were covered with a thin layer of wax which was liquefied at a temperature of $70^{\circ} \mathrm{C}$ and applied on the roots with a paint brush to provide a layer simulating periodontal membrane [13], then the roots were mounted vertically in selfcure acrylic resin blocks, exposing $10 \mathrm{~mm}$ of the coronal and middle parts. Acrylic blocks together with the teeth were placed on the lower fixed compartment of the Instron Universal Testing Machine and a compressive vertical loading was applied by the Universal Testing Machine with spherical tips of $0.5 \mathrm{~mm}$ diameter at a speed of $1 \mathrm{~mm} / \mathrm{min}$ on the coronal cut surfaces of the teeth until fracture of the specimens occur (Figure 1C).

The force at which the fracture occurred was recorded in Newtons and the data was analyzed using One-way ANOVA test followed by Tukey post hoc test for pairwise comparisons.

\section{Assessment of mode of fracture}

Based on a study by Ulusoy., et al. (2016) and Alharbi., et al. (2014) $[15,16]$, for assessing the mode of fracture, Teeth were then examined after fracture using a stereomicroscope and classified according to the type of the fracture into supracrestal root fracture in which the root fractures above the level of acrylic resin, subcrestal root fracture, in which the root fractures below the level of acrylic resin and vertical root fracture, in which the fracture line extends along the long axis of the tooth and Categorical data were presented as frequencies and percentages and were analyzed using Fisher's exact test.

\section{Statistical analysis}

Data were tested for normality using Shapiro Wilk test, Continuous data were presented as mean and standard deviation and were statistically analyzed using One-way ANOVA test followed by Tukey post hoc test for pairwise comparisons.

Categorical data were presented as frequencies and percentages and were analyzed using Fisher's exact test.

Independent $\mathrm{t}$ test was used for two groups comparison. A pvalue less than or equal to $0.05(p \leq 0.05)$ was considered statistically significant.

\section{Results}

The means, standard deviations, as well as the minimum and maximum values of the fracture resistance test are shown in table 1 , and the results of Tukey post hoc test for pairwise comparison of fracture resistance are shown in table 2.

\begin{tabular}{|l|c|c|c|c|c|c|}
\hline & $\begin{array}{c}\text { Group I } \\
\text { (Biodentine) }\end{array}$ & $\begin{array}{c}\text { Group II } \\
\text { (RetroMTA) }\end{array}$ & $\begin{array}{c}\text { Group III } \\
\text { (PC) }\end{array}$ & $\begin{array}{c}\text { Group IV } \\
\text { (Positive control) }\end{array}$ & $\begin{array}{c}\text { Group V } \\
\text { (Negative control) }\end{array}$ & P - Value \\
\hline Mean & 333.25 & 279.51 & 293.31 & 276.39 & 590.05 & $<0.001^{*}$ \\
\hline SD & 63.17 & 102.96 & 71.50 & 127.68 & 139.58 & \\
\hline Minimum & $268.55 \mathrm{~N}$ & $149.41 \mathrm{~N}$ & $172.58 \mathrm{~N}$ & $132.84 \mathrm{~N}$ & $382.93 \mathrm{~N}$ & \\
\hline Maximum & $400.73 \mathrm{~N}$ & $391.29 \mathrm{~N}$ & $358.13 \mathrm{~N}$ & $495.72 \mathrm{~N}$ & $755.56 \mathrm{~N}$ & \\
\hline
\end{tabular}

Table 1: Mean, standard deviation (SD), minimum and maximum values of fracture resistnace (in Newtons) for the experimental and the control groups.

\begin{tabular}{|l|c|c|c|c|c|}
\hline & $\begin{array}{c}\text { Group I } \\
\text { (Biodentine) }\end{array}$ & $\begin{array}{c}\text { Group II } \\
\text { (RetroMTA) }\end{array}$ & $\begin{array}{c}\text { Group III } \\
\text { (PC) }\end{array}$ & $\begin{array}{c}\text { Group IV } \\
\text { (positive control) }\end{array}$ & $\begin{array}{c}\text { Group V } \\
\text { (Negative control) }\end{array}$ \\
\hline Group I (Biodentine) & $\mathrm{X}$ & 0.9 & 0.964 & 0.881 & $0.002^{*}$ \\
\hline Group II (RetroMTA) & $\mathrm{X}$ & $\mathrm{X}$ & 0.999 & 1 & $<0.001^{*}$ \\
\hline Group III (PC) & $\mathrm{X}$ & $\mathrm{X}$ & $\mathrm{X}$ & 0.999 & $<0.001^{*}$ \\
\hline Group IV (Positive control) & $\mathrm{X}$ & $\mathrm{X}$ & $\mathrm{X}$ & $\mathrm{X}$ & $<0.001^{*}$ \\
\hline Group V (Negative Control) & $\mathrm{X}$ & $\mathrm{X}$ & $\mathrm{X}$ & $\mathrm{X}$ & $\mathrm{X}$ \\
\hline
\end{tabular}

Table 2: Results of Tukey post hoc test for pairwise comparison of fracture resistance.

*: Significant at $\mathrm{p}<0.05$. 
There was a statistically significant difference among the five groups $(\mathrm{p}<0.001)$. The highest mean fracture resistance value was reported to Group V (negative control group): $590.05 \pm 139.58$ with statistical significant difference when compared to other experimental groups $(\mathrm{p}<0.001)$. Among the 3 experimental groups, the highest mean fracture resistance value was reported to Group I (Biodentine): $333.25 \pm 63.17$ followed by Group III (Portand Cement): $293.31 \pm 71.50$ and Group II (RetroMTA): $279.51 \pm 102.96$ respectively with no statistical significant difference among them ( $p>0.05)$ and the lowest mean fracture resistance value was reported to Group IV (positive control group): $276.39 \pm 127.68$ with no statistical significance difference $(p>0.05)$.
There was no statistically significant difference regarding the mode of fracture between the five groups ( $p=0.057)$ as shown in table 3. Group I (Biodentine) showed 28.6\% supracrestal fracture, $14.3 \%$ subcrestal fracture and $57.1 \%$ vertical root fracture. Group II (RetroMTA) showed 57.1\% supracrestal fracture, 14.3\% subcrestal fracture and $28.6 \%$ vertical root fracture. Group III (Portland Cement) showed 71.4\% supracrestal fracture, 0.0\% subcrestal fracture and $28.6 \%$ vertical root fracture. Group IV (Positive control, instrumented roots but not filled) showed $85.7 \%$ supracrestal fracture, $0.0 \%$ subcrestal fracture and $14.3 \%$ vertical root fracture. Group V (Negative Control, decoronated intact roots) showed $42.9 \%$ supracrestal fracture, $57.1 \%$ subcrestal fracture and $0.0 \%$ vertical root fracture.

\begin{tabular}{|c|c|c|c|c|c|c|c|c|c|c|c|}
\hline \multirow[t]{2}{*}{ Type of fracture } & \multicolumn{2}{|c|}{ Group I Biodentine } & \multicolumn{2}{|c|}{$\begin{array}{c}\text { Group II } \\
\text { RMTA }\end{array}$} & \multicolumn{2}{|c|}{$\begin{array}{c}\text { Group III } \\
\text { PC }\end{array}$} & \multicolumn{2}{|c|}{ Group IV } & \multicolumn{2}{|c|}{ Group V } & \multirow[t]{2}{*}{ P-Value } \\
\hline & $\mathbf{N}$ & $\%$ & $\mathbf{N}$ & $\%$ & $\mathbf{N}$ & $\%$ & $\mathbf{N}$ & $\%$ & $\mathbf{N}$ & $\%$ & \\
\hline Supracrestal & 2 & $28.6 \%$ & 4 & $57.1 \%$ & 5 & $71.4 \%$ & 6 & $85.7 \%$ & 3 & $42.9 \%$ & \\
\hline Subcrestal & 1 & $14.3 \%$ & 1 & $14.3 \%$ & 0 & $0.0 \%$ & 0 & $0.0 \%$ & 4 & $57.1 \%$ & 0.057 \\
\hline Vertical & 4 & $57.1 \%$ & 2 & $28.6 \%$ & 2 & $28.6 \%$ & 1 & $14.3 \%$ & 0 & $0.0 \%$ & \\
\hline Total & 7 & $100 \%$ & 7 & $100 \%$ & 7 & $100 \%$ & 7 & $100 \%$ & 7 & $100 \%$ & \\
\hline
\end{tabular}

Table 3: Frequencies, percentages and the results of Fisher's exact test for comparison of mode of fracture between the five groups.

\section{Discussion}

Teeth with internal root resorption have thin root canal walls causing the roots to be more liable to fracture. Thus, materials that have reinforcing capacity are better to be used for filling the resorptive areas with thin dentinal walls to avoid fractures. The aim of the present study was to evaluate the reinforcing effect of Portland cement, RetroMTA and Biodentine in repairing simulated perforating internal root resorption defects on the fracture resistance of the teeth.

In this study mandibular premolars were used as they simulate the clinical situation better where chewing forces are maximum. Also, they have a circular cross-section in the mid to the apical region that allow for uniform distribution of load to fracture [14]. Canals were mechanically instrumented using Revo-S rotary files, this rotary system shows asymmetric cross-sectional geometry aiming to decrease the stress during root canal preparation [17].

To simulate the periodontal ligament, the apical $5 \mathrm{~cm}$ of the teeth was covered by softened pink wax to prevent stress concentration in one particular region and transfers the stresses produced by load application all over the root surface [14]. Though removal or retention of smear layer before canal obturation still remains controversial, yet in the present study, 17\% EDTA was used to remove the smear layer before obturating the canals and filling the resorptive cavities to improve the sealing capability of the filling materials [14].

Simulated perforating internal resorption defects were created in the corono-middle part of the root to facilitate their manipulation and cavities with a smooth outline using round burs were done to ensure standardization among all the groups. Using round burs to simulate the resorption defects may result in a resorption cavity which does not replicate a real unformed, irregular resorption cavity. However, standardizing the cavity dimension in all the study groups makes it applicable to compare [2].

Studies displayed different orientation of applied forces, however mandibular posterior teeth sustain vertical forces more than lateral forces during root canal obturation and occlusion, thus we used vertical forces of loading angle $90^{\circ}$ to test the fracture resistance. Although the applied force could not completely simulate 
the clinical situations but standardizing the force in all the study groups makes it possible to compare the reinforcing ability of the materials tested $[2,15]$.

According to the results of this study, Fracture resistance values of teeth repaired with Biodentine 333.25 \pm 63.17 were higher than those of RetroMTA $279.51 \pm 102.96$ and Portland cement 293.31 \pm 71.50 without a statistical significant difference $(\mathrm{p}>0.05)$. This could be reported to that Biodentine release larger amounts of calcium which may lead to the higher formation of interfacial layer and tag-like structures which increase the bonding ability of Biodentine. It was suggested that calcium silicate-based materials chemically bond to root canal dentine, although the positive correlation between bond strength and fracture resistance is not clear, it was accepted that successful adhesion of the materials to the root dentine increases their reinforcing effect [15].

Also, Biodentine exhibits a very good marginal adaptation which could be due to its smaller particle size, as exhibited by the greater specific surface area of Biodentine $\left(2.811 \mathrm{~m}^{2} / \mathrm{g}\right)$ as mentioned in the study made by Lertmalapong., et al. (2018), A high specific surface area promotes water adsorption and hydration reactions of bioceramics, resulting in a greater expansion of Biodentine [18].

A study by Vipin., et al. 2013 [19], reported that there is a sharp increase in the compressive strength reaching more than $100 \mathrm{MPa}$ in the first hour. The mechanical strength continues to improve to reach more than $200 \mathrm{MPa}$ at $24 \mathrm{~h}$ which is more than most glass ionomers values. A specific feature of Biodentine is its capacity to continue improving with time over several days until reaching 300 MPa after one month. This value becomes quite stable and is in the range of the compressive strength of natural dentin (297 MPa). This maturation process can be related to the decrease of porosity with time. The material properties of Biodentine are similar to those of dentin. All of the modulus of elasticity of the cement and also the pressure resistance, bending strength and Vickers hardness are comparable to that of the dentin [19].

Biodentine is comprised of calcium silicate which expands by $0.2 \%-6 \%$ of its initial volume. This expansion greatly contributes to its sealing capacity and is responsible for the highest fracture resistance values observed in the Biodentine group [18].

Portland cement showed relatively high fracture resistance values, this could be attributed to that when PC is hydrated it initially forms calcium hydroxide and calcium silicate hydrate gel that fi- nally change into a poorly porous solid and crystallized gel [20]. Barium sulphate is added as a radiopacifying agent which possess a very high specific surface area as reported by Camilleri and Gandolfi 2010 [11]; thus achieving excellent workability and material strength.

It was shown that the materials with similar elastic modulus to dentin could reinforce the weak roots. The elastic modulus of Portland cements is around 15 - $30 \mathrm{GPa}$ and dentin which is about 14 - 18.6 GPa and for Biodentine is $22.0 \mathrm{GPa}$, and thus, the reinforcing effect of portland cement and biodentine may be explained by their similar elastic modulus to dentin [14].

The group of teeth repaired with RetroMTA showed the lowest fracture resistance values compared to the other groups. This could be due to the low push out strength of the RetroMTA as mentioned in a previous study made by Orhan., et al. 2019 who also claimed that when mixing RetroMTA with the instructed amount of water, RetroMTA appeared dry which made it relatively difficult to condense to the cavity like holes [21].

Although TCSs that hydrate do not require a precise ratio, inconsistent powder/liquid ratio, insufficient condensing or dehydration may cause macro and micro porosities which can lead to leakage. If not all powder are hydrated during mixing, the ultimate strength of the material could be reduced and this could be one of the reasons why RetroMTA revealed the least fracture resistance value [21].

The low expansion coefficient of RetroMTA (0.09\% volume) might have generated a large gap leading to its inferior results same as in the study conducted by Lertmalapong., et al. (2018). Lertmalapong claimed that RetroMTA showed high mean percentages of gap areas in comparison to other materials [18].

The mean fracture resistance values among the experimental groups did not show a statistical significant difference, this could be due to the lack of studies concerning the fracture resistance of teeth with internal perforating root resorption repaired with different calcium silicate cements [2]. Also the sample size may have contributed to the ability to detect such small differences statistically because of the precision of the variables being measured [22].

Positive control group showed the least mean fracture resistance values, although it was not statistically significant. The explanation of the enhanced fracture resistance of the experimental 
groups compared with the positive control group could be attributed to the development of a hydroxyapatite-like layer between dentin and tricalcium silicate cements through the hydration reaction of TCSMs in the presence of synthetic tissue fluid (i.e. PBS). The formation of a hybrid layer between TCSMs and dentin suggests that chemical bonding has been formed thus enhancing their fracture resistance [23].

The majority of the fractures after loading were vertically or obliquely oriented and mainly located above the level of acrylic resin base (supracrestal root fracture). This could be attributed to the location of the resorption cavities with fragile dentinal walls are found in the corono-middle third of the roots and did not extend below the acrylic resin block [15].

The reason that Group V (negative control, decoronated intact roots) showed the highest percentage of subcrestal fracture could be attributed to that these teeth tends to transfer the stress concentration to the root apex. This behaviour may also explain the reason of the high fracture resistance observed in Group V and the prevalence of the subcrestal type of fracture, thus showing that the fracture resistance does not correlate with the fracture mode [24].

The higher incidence of vertical root factures observed in Group I (Biodentine) could be correlated with the elastic modulus of the biodentine material. When the force was applied stresses were apparently concentrated on the coronal facial third and apical lingual sides of the root. When the tooth could not tolerate the applied force, failure occurred at the weakest area of the root causing vertical fracture [16].

Supracrestal fracture of teeth could be attributed to low modulus of elasticity of the materials where the crown could not withstand and coronal fractures occur. There are different and many parameters which affect the fracture resistance reported in different studies including the dimensions of the selected teeth, amount of tooth structure removed and remaining after a tooth preparation and the distance between crown margins and level of the base supporting specimens during testing [16].

\section{Conclusion}

Within the limitations of this study, it was concluded that, filling teeth with internal root resorption defects using a hybrid technique, where the apical part of the root canal is filled with gutta-percha and the resorption area is filled with a biocompatible material enhances the reinforcing capacity of the weakened roots and avoid their fracture and several bioceramic materials such as biodentine have a reinforcing effect and can be used as an alternative to MTA in repairing resorptive defects and perforations.

\section{Conflict of Interest}

There is no conflict of interest in the study.

\section{Bibliography}

1. Nilsson E., et al. "Management of Internal Root Resorption on Permanent Teeth". International Journal of Dentistry (2013): $1-7$.

2. Türker S., et al. "Fracture Resistance of Teeth with Simulated Perforating Internal Resorption Cavities Repaired with Different Calcium Silicate-based Cements and Backfilling Materials". Journal of Endodontics 44.5 (2018): 860-863.

3. Estrela C., et al. "Root perforations: a review of diagnosis, prognosis and materials". Brazilian Oral Research (2018) 32.

4. De-Deus G., et al. "The Ability of Portland Cement, MTA, and MTA Bio to Prevent Through-and-Through Fluid Movement in Repaired Furcal Perforations". Journal of Endodontics 33.11 (2007): 1374-1377.

5. Borges ÁH., et al. "Portland Cement Use in Dental Root Perforations: A Long Term Followup". Case Reports in Dentistry (2014): 1-5

6. Raghavendra SS., et al. "Bioceramics In Endodontics - A Review". Journal of Istanbul University Faculty of Dentistry 51 (2017).

7. Pornamazeh T., et al. "In Vitro Cytotoxicity and Setting Time Assessment of Calcium-Enriched Mixture Cement, Retro Mineral Trioxide Aggregate and Mineral Trioxide Aggregate". The Iranian Endodontic Journal 12.4 (2017): 488-492.

8. Souza IC De., et al. "Analysis of radiopacity, $\mathrm{pH}$ and cytotoxicity of a new bioceramic material". Journal of Applied Oral Science 23.4 (2015): 383-389.

9. Tirone F., et al. "Response of periodontium to mineral trioxide aggregate and Biodentine: a pilot histological study on humans". Australian Dental Journal 63.2 (2018): 231-241.

10. Sinkar RC., et al. "Comparison of sealing ability of ProRoot MTA, RetroMTA, and Biodentine as furcation repair materials: 
An ultraviolet spectrophotometric analysis". Journal of Conservative Dentistry 18.6 (2015): 445-448.

11. Camilleri J. "Evaluation of the physical properties of an endodontic Portland cement incorporating alternative radiopacifiers used as root-end filling material". International Endodontic Journal 43.3 (2010): 231-240.

12. Menezes R., et al. "Microscopic analysis of dog dental pulp after pulpotomy and pulp protection with mineral trioxide aggregate and white Portland cement". Journal of Applied Oral Science 12.2 (2004): 104-107.

13. Menezes R., et al. "Histologic evaluation of pulpotomies in dog using two types of mineral trioxide aggregate and regular and white Portland cements as wound dressings". Oral Surgery, Oral Medicine, Oral Pathology, Oral Radiology, and Endodontology 98.3 (2004): 376-379.

14. Girish K., et al. "Effect of obturating materials on fracture resistance of simulated immature teeth". Journal of Conservative Dentistry: JCD 20.2 (2017): 115-119.

15. Ulusoy Öİ and Paltun YN. "Fracture resistance of roots with simulated internal resorption defects and obturated using different hybrid techniques". The Journal of Dental Sciences 12.2 (2017): 121-125

16. Alharbi F., et al. "Fracture resistance and failure mode of fatigued endodontically treated teeth restored with fiber-reinforced resin posts and metallic posts in vitro". Dental Traumatology 30.4 (2014): 317-325.

17. Basrani B., et al. "Torsional Profiles of New and Used Revo-S Rotary Instruments: An In Vitro Study". Journal of Endodontics 37.7 (2011): 989-992.

18. Lertmalapong P., et al. "Bacterial leakage and marginal adaptation of various bioceramics as apical plug in open apex model". Journal of Investigative and Clinical Dentistry (2018): e12371.

19. Vipin A., et al. "Bioactive dentin replacement". IOSR Journal of Dental and Medical Sciences 12.4 (2013): 51-57.

20. Nashaat YM and Roshdy NN. "Comparative Evaluation of the Push-out bond strength of Nano-formulations of MTA and Portland cement". Egyptian Dental Journal 66.4 (2020): 28072814.
21. Orhan EO., et al. "Evaluation of the bond strengths of two novel bioceramic cement using a modified thin-slice push-out test model". International Journal of Applied Ceramic Technology 16.5 (2019): 1998-2005.

22. Williams C., et al. "A Comparison of Cohesive Strength and Stiffness of Resilon and Gutta-Percha". Journal of Endodontics 32.6 (2006): 553-555.

23. Elnaghy AM and Elsaka SE. "Fracture resistance of simulated immature teeth filled with Biodentine and white mineral trioxide aggregate - an in vitro study". Dental Traumatology 32.2 (2016): 116-120.

24. Castro CG., et al. "Fracture resistance and mode of failure of various types of root filled teeth". International Endodontic Journal 45.9 (2012): 840-847.

Volume 5 Issue 8 August 2021

(C) All rights are reserved by Paunceh Samy Soliman., et

al. 